\title{
Delay-Optimized File Retrieval under LT-Based Cloud Storage
}

\author{
Haifeng Lu,Chuan Heng Foh, Senior Member, IEEE, Yonggang Wen, Member, IEEE, \\ and Jianfei Cai, Senior Member, IEEE
}

\begin{abstract}
Fountain-code based cloud storage system provides reliable online storage solution through placing unlabeled content blocks into multiple storage nodes. Luby Transform (LT) code is one of the popular fountain codes for storage systems due to its efficient recovery. However, to ensure high success decoding of fountain codes based storage, retrieval of additional fragments is required, and this requirement could introduce additional delay. In this paper, we show that multiple stage retrieval of fragments is effective to reduce the fileretrieval delay. We first develop a delay model for various multiple stage retrieval schemes applicable to our considered system. With the developed model, we study optimal retrieval schemes given requirements on success decodability. Our numerical results suggest a fundamental tradeoff between the file-retrieval delay and the target probability of successful file decoding, and that the file-retrieval delay can be significantly reduced by optimally scheduling packet requests in a multi-stage fashion.
\end{abstract}

Index Terms-Delay-optimal retrieval, LT codes, Cloud storage.

\section{INTRODUCTION}

Cloud storage systems provide a scalable online storage solution to end users who require flexible amount of storage space but do not wish to own and maintain storage infrastructure [1], [2]. Compared with traditional data storage, cloud storage has several advantages. For example, end users can access their data anywhere through Internet without bothering about carrying physical storage media. Also, different users can collaboratively contribute to the data stored in cloud storage with permission from the data owner. Due to its high popularity in industry, cloud storage has been a hot topic in cloud computing community [3].

Generally, cloud storage systems rely on thousands of storage nodes. A content is often fragmented and distributed into a set of storage nodes. To offer high reliability and availability of storage services, redundancy of contents may be employed. Fragments of

Haifeng Lu is with Alibaba Inc., China (email: haifeng.Ihf@alibabainc.com).

Chuan Heng Foh is with the Institute for Communication Systems Research, University of Surrey, Guildford, Surrey, GU2 7XH, UK (email:c.foh@surrey.ac.uk).

Yonggang Wen and Jianfei Cai are with the School of Computer Engineering, Nanyang Technological University, 639798, Singapore (email:ygzwen@ntu.edu.sg, asjfcai@ntu.edu.sg). contents may be simply replicated and stored in different storage nodes to achieve redundancy. Other than naive replication, Lin et al. [4] proposed two QoSaware data replication algorithms to reduce storage cost while maintaining QoS for the applications. In [5], Weatherspoon et al. analyzed two schemes for redundancy: replication and erasure coding, concluding that erasure-coded systems can provide higher availability with lower bandwidth and less storage space. Since then, there are plenty of works on designing erasure codes for storage systems, and they mainly focus on the reliability and availability of storage systems [6], [7], [8], [9].

Recently, motivated by the great success of rateless codes or fountain codes, which have very low decoding complexity and can generate infinite number of encoded packets, some works [10], [11] have applied the popular rateless code or LT code, into cloud storage systems and have achieved promising performance. The main advantage for a rateless code based cloud storage system is that it significantly simplifies the challenging content placement and content recovery problems that need to be addressed in erasure code based systems. This is because a rateless code based system can potentially generate infinite number of encoded packets to be placed across the storage system and to replace those unavailable packets due to node failure. However, the disadvantage of a rateless code based cloud storage system is that it incurs longer data retrieval delay, since it requires more coded packets due to its uncertainty in decoding different from its erasure code counterpart which is deterministic in decoding. Therefore, in this paper we focus on designing an optimized retrieval method for an LT code based cloud system. As stated in [12], traffic hot spots emerge at the core of a cloud storage system which is responsible for communications between the system and end users. We call this core as a portal. In this research, we focus on addressing the problem how to minimize the retrieval delay generated by the portal in an LT-based cloud storage system.

In particular, unlike the Maximum Distance Sepa- 
rable (MDS) codes such as Reed-Soloman code [13], which need deterministic number of redundant encoded packets for decoding, a storage collector operating LT decoder requires an uncertain number of encoded fragments from the storage system for successful decoding. These uncertain number of encoded packets add delay in storage retrieval. Thus, a tradeoff between the successful decoding probability and storage retrieval delay exists. In this paper, we study such a tradeoff and propose a multiple-stage storage retrieval scheme where we show that storage retrieval delay can be reduced without sacrificing the performance on decoding probability. We further demonstrate the optimal retrieval setup for the case of a two-stage retrieval.

The contributions of this paper are twofold. Firstly, we develop a model describing the multi-stage request scheme for an LT code based storage system. Secondly, we solve the optimization problem for the optimal two-stage request scheme and provide a closed-form expression for analysis.

The remainder of this paper is organized as follows. In Section 2, we briefly review the related work. In Section 3, we introduce the system architecture and present the formal definition of the optimal retrieval delay problem. A detailed description of LT codes and its decodability is presented in Section 4. Section 5 presents a rigorous analysis on the delay for different retrieval schemes together with simulation validation. Section 6 provides analysis on the optimal two-stage request scheme. We then demonstrate the tradeoff between the delay and the probability of successful decoding in Section 7. We also show some simulation results using different traffic models in Section 8. Finally, some important conclusions are drawn in Section 9.

\section{Related Work}

Erasure code based cloud storage systems: An example of a successful deployment of commercial cloud storage system using erasure coding is Symform [14], [15]. In Symform, Reed-Solomon code [13] is used for redundancy generation. Its success shows erasurecode-based cloud storage systems are practical and comparable with traditional replication-based cloud storage systems. Another research effort focuses on the design of storage system operational optimization based on existing erasure codes to achieve some specific optimal performance matrices. These operational designs may include storage redundancy overhead optimization, storage allocation and repair, and others [16], [17], [18], [19]. As aforementioned, compared with erasure code based cloud storage systems, LT code based systems simplify content placement and recovery problems at the cost of longer retrieval delay.

LT code: Luby Transform (LT) code [20] enjoys relatively low computational complexity of $O(k \ln (k / \delta))$ for recovering $k$ symbols with $O\left(\sqrt{k} l n^{2}(k / \delta)\right)$ additional packets. Here, $1-\delta$ is the probability of successful recovery of all $k$ symbols. The property of low decoding complexity makes LT code suitable for end users equipped with moderate CPU. Another property of LT code is rateless which means the redundancy ratio of data can be arbitrarily changed without additional design. This property is naturally fit with cloud storage systems with thousands of storage nodes storing various type of data. The promising performance of LT code based storage systems has been shown in [10], [11].

Data retrieval performance in storage systems: In addition to the reliability and availability issues of a storage system, another important problem raised in a storage system is data retrieval performance. Traditional distributed file systems like NFS [21] and Sprite [22] are single server based and achieve acceptable retrieval performance through file caching. However, the retrieval performance is bounded by the speed of the single storage server. Conversely, parallel file systems, such as Vesta [23], Galley [24], PVFS [25] and GPFS [26], allow data spreading across multiple storage nodes and provide parallel access to it. The high transfer rate is reached by various techniques such as I/O scheduling, content prefetching, etc. More recently, high retrieval performance is achieved by introducing fast storage devices like solid-state drivers (SSD) [27] and DRAM [28]. Unlike these existing work dealing with the retrieval performance problem from the application level, we consider this problem from the network level since in an LT code based storage system the probabilistic LT decoding could result in large retrieval delay due to decoding failure. In [29], [30], [31], [32], the authors discussed some mechanisms for reducing data fetching latency in distributed networks. Those mechanisms are designed for general content without considering any coding metrics, and they could be applied together with our method.

\section{System Model and Problem state- MENT}

In this section, we first present a schematic description of a cloud storage architecture, in which LT-encoded data packets are spread out across a pool of storage nodes for high availability. Following that, we formulate a delay-optimal file-retrieval problem, which aims to minimize the retrieval delay by strategically scheduling packet retrieval requests. The notations used in this section are summarized in Table 1.

\subsection{System Architecture}

In this paper, we focus on a distributed cloud storage system (e.g., a community-based cloud storage system as in Tahoe-LAFS [33]). As illustrated in Figure 1, the cloud storage system manages a set of storage 
This article has been accepted for publication in a future issue of this journal, but has not been fully edited. Content may change prior to final publication. Citation information: DOI 10.1109/TCC.2015.2430347, IEEE Transactions on Cloud Computing

TABLE 1

Notations

\begin{tabular}{|l|l|}
\hline Notation & Description \\
\hline$k$ & No. of original symbols. \\
\hline$n$ & $\begin{array}{l}\text { No. of LT encoded symbols for } \\
\text { certain target decoding probability. }\end{array}$ \\
\hline$\lambda_{a}$ & Arrival rate for ambient traffic. \\
\hline$l, \sigma^{2}$ & $\begin{array}{l}\text { Mean and variance of length of } \\
\text { ambient traffic packets. }\end{array}$ \\
\hline$\theta$ & Arrival rate for LT traffic. \\
\hline$l_{L T}$ & Length of LT encoded symbols. \\
\hline$t_{i}$ & Arrival time for $i$ th LT encoded symbol. \\
\hline$s$ & No. of stages. \\
\hline$n_{i}$ & $\begin{array}{l}\text { No. of LT encoded symbols requested } \\
\text { in stage } i .\end{array}$ \\
\hline
\end{tabular}

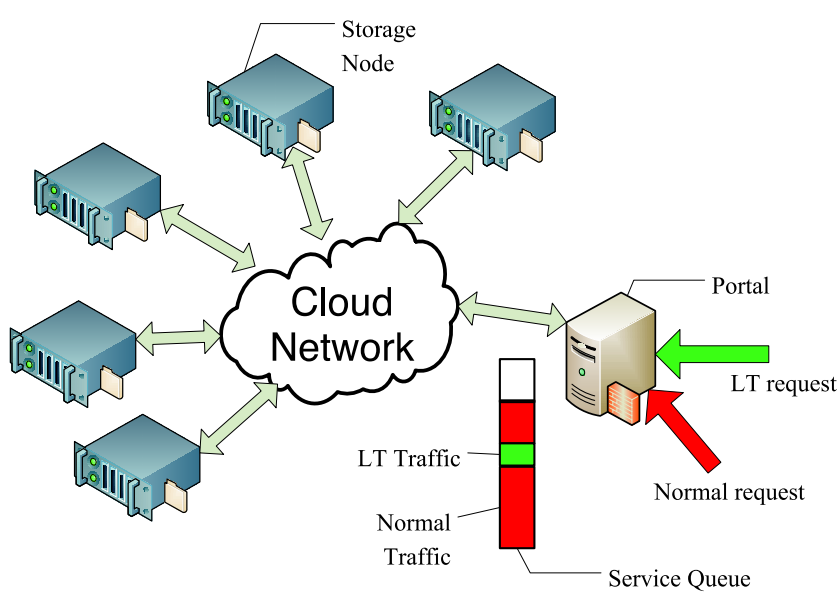

Fig. 1. System architecture for a cloud storage system.

nodes through a communication network. The users can access to the storage service through a dedicated portal. One particular storage application of interest in this research is the file storage.

We assume that the storage service provides both premium LT-based file storage and regular file storage. For the LT-based file storage, the portal, upon receiving a file from a user request, encodes the file with a chosen LT code into a set of LT fragments or packets ${ }^{1}$ and spreads them across different nodes for reliability and high availability. Upon receiving a fileretrieval request, the portal sends a few packet retrieval requests to different storage nodes, and gathers sufficient encoded packets to be forwarded to the user. For the regular file storage, the portal simply stores the received user file into a chosen storage node, with some potential replication in another set of nodes.

We assume that the number of subscribers for the premium LT-based storage service is much smaller than that for the regular storage service. As such, it is with high probability that, at one instant, only one user will retrieve his/her LT-coded files and the majority of the file-retrieval requests are for the regular storage service. In this paper, we will analyze such a

1. Here we use the term packet interchangeably with fragment as we assume that an IP packet carries an LT encoded fragment in the system.

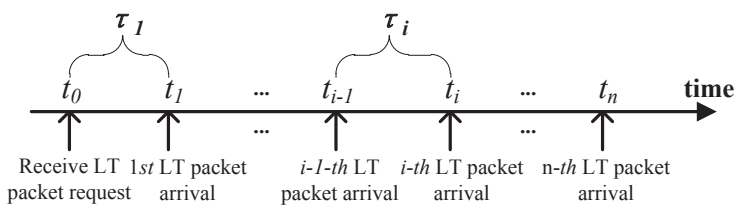

Fig. 2. Arrival process of LT encoded packets seen at the portal for a file-retrieval request.

simplified use case. The insights obtained through the mathematical framework and numerical analysis will shed lights on more practical application scenarios.

\subsection{Traffic Model}

In this architecture, the portal node observes two types of packets. The first one is packets for the regular storage service, considered as a process of ambient traffic. The ambient traffic follows a particular arrival process with an arrival rate of $\lambda_{a}$. Moreover, the length of ambient packets is modeled as a random variable with mean $l$ and variance $\sigma^{2}$. The second one is the LT-coded packets for the premium storage service. We assume the arrival delay of each LT-coded packet is identically distributed, each of which is a certain random variable with a mean value of $\theta^{-1}$. The length of LT-coded packets is assumed to be a constant, denoted by $l_{L T}$.

When the portal requests $n$ LT-coded packets from the storage grid, the packet arrival process is illustrated in Figure 2. We assume that the packet request is sent out at time 0 (i.e., $t_{0}=0$ ), and denote the arrival time of the $i$-th LT-coded packet as $t_{i}$. We also denote the inter-arrival time between the $i-1$-th packet and the $i$-th packet as $\tau_{i}=t_{i}-t_{i-1}$.

\subsection{Problem Statement}

In the considered distributed storage system, the bottleneck lies on the portal, because a large number of users can issue their file requests to the portal. In this paper, we focus on the file-retrieval delay, defined as the duration between the time for the portal receiving an LT-coded file request and the time when the last LT-coded packet is sent out by the portal. The fileretrieval delay is a good indicator of user experience. Therefore, we aim to reduce the file-retrieval delay by strategically scheduling the LT-coded packet requests.

The delay-optimal file-retrieval problem is stated as follows. We assume a probabilistic file-retrieval model. In order to achieve the probability of successful decoding of $p$, the portal needs to request $n$ LT encoded packets from the storage grid. Traditionally, the $n$ packets are requested in one shot. This scheme is referred to as a one-stage request scheme. In this paper, we propose to use a multiple-stage request scheme to improve the file-retrieval performance. Specifically, the user can divide the request into $s$ stages, each consisting of requests for $n_{1}, n_{2}, \cdots, n_{s}$ packets $\left(\sum_{i} n_{i}=n\right)$. 
If the user successfully decodes the file after stage $m$, it will stop requesting the rest $\sum_{i=m+1}^{s} n_{i}$ packets.

In our proposed multi-stage request scheme, the designing objective is to minimize the average fileretrieval delay, for a given number of stages. Mathematically, it can be formulated as the following optimization problem,

$$
\begin{array}{ll}
\min & \bar{D}_{s}\left(n_{1}, n_{2}, \cdots, n_{s}\right) \\
\text { s.t. } & \sum_{i=1}^{s} n_{i}=n, \\
& n_{i} \geq 0,1 \leq i \leq s .
\end{array}
$$

Here $\bar{D}_{s}(\cdot)$ denotes the average retrieval delay under a given request scheme.

Intuitively, multiple stages of packet retrieval incurs additional delay in storage retrieval. However, we will show that the multi-stage retrieval scheme can outperform the single-stage retrieval scheme in terms of the average file-retrieval delay. This delay reduction originates from the following observation. In the multi-stage retrieval scheme, just retrieving an adequate number of packets may already give sufficiently high success decodability, eliminating the need for further rounds of packet retrieval. Only when the file cannot be decoded with the set of received packets, the additional packet requests are sent in subsequent stages. In such a case, the single-stage retrieval scheme is a special case of our proposed multi-stage retrieval scheme, when $s=1$. From an optimization perspective, the performance of the multistage retrieval scheme cannot be worse than that of the single-stage scheme.

\section{LT Codes AND ITS DECodability}

In this section, we present a detailed description about LT-codes, including its encoding and decoding processes, and the probability of decoding files for a given number of retrieved packets.

\subsection{LT Encoder/Decoder}

LT codes are the first class of digital fountain codes, with nearly optimal erasure correcting capability. Its main characteristic lies in employing a simple algorithm based on the exclusive-or operation to encode and decode the message. As such, it is particularly appealing to cloud-based file storage. In the LT-based cloud storage system, a file is first broken into $k$ source symbols, and then these $k$ source symbols are encoded into $n$ packets and spread across a pool of storage nodes. The number of encoded packets $n$ can be arbitrary large depending on the duplication ratio of the system. In this subsection, we briefly explain its encoding and decoding processes.

The encoding and decoding processes of LT codes can be illustrated via an example, as shown in Figure 3.
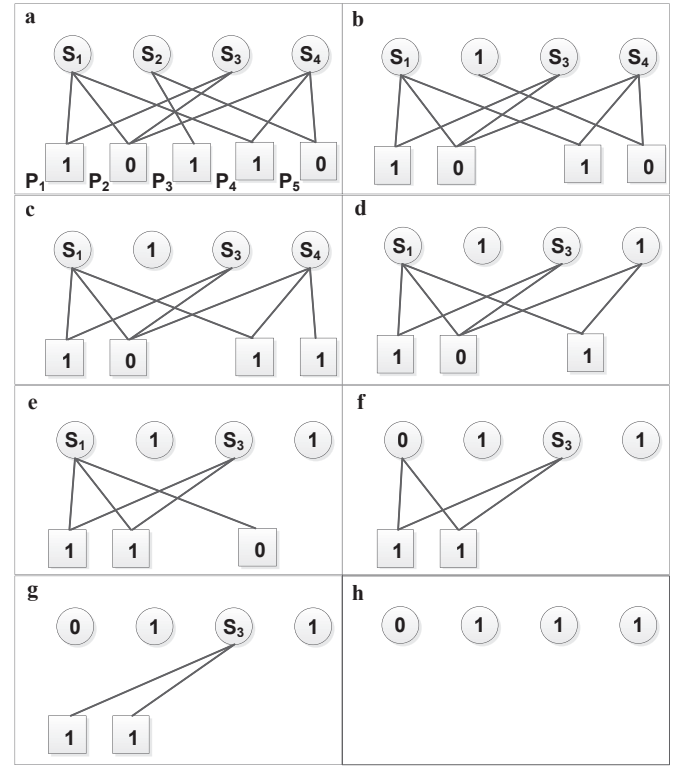

Fig. 3. An example of LT encoding and decoding procedures for $k=4$ and $n=5$.(a) LT encoding: encode 4 source symbols, $S_{1}-S_{4}$, into 5 packets, $P_{1}-P_{5}$. (b)-(h) LT decoding to recover the 4 source symbols.

An LT encoder follows a three-step process to generate one encoded packet. First, a degree $d \in[1, k]$ is randomly chosen from a degree distribution of $\Omega_{d}$. Second, $d$ distinct source symbols are chosen, uniformly at random, from the set of source symbols. Finally, the encoded packet is equal to the bitwise sum, modulo 2 , of those $d$ chosen source symbols. The information about the set of chosen source symbols is called coding vector and it is recorded in the head of the encoded packet. The same process repeats until $n$ encoded packets are generated. In Figure 3, Panel (a) demonstrates an example of LT encoding procedure with 4 binary source symbols (i.e. $k=4$ ), denoted by $S_{1}, S_{2}, \cdots, S_{4}$, and 5 LT encoded packets (i.e. $n=5$ ), denoted by $P_{1}, P_{2}, \cdots, P_{5}$ with value 10110 .

The LT decoding process employs a simple message passing algorithm [34], which iteratively decodes source symbols from degree- 1 encoded packets. Consider the same example illustrated in Figure 3. At the first iteration, the only encoded packets with degree equal to 1 is $P_{3}$ (refer to Panel (a)). Thus, the source symbol $S_{2}$ which is connected to $P_{3}$ is set to 1 (refer to Panel (b)). After $S_{2}$ is recovered, the encoded packets which have connections with $S_{2}$ are extracted by the value of $S_{2}$ and the connections are removed (refer to Panel (c)). After this step, a new symbol $S_{4}$ can be recovered and the same decoding procedure is executed iteratively until no more degree- 1 encoded packets can be found. If there are still some encoded packets which are not recovered, a decoding failure is reported. In order to recover the rest of source symbols, one can request more encoded packets. Clearly, the LT decoding process cannot guarantee to recover 


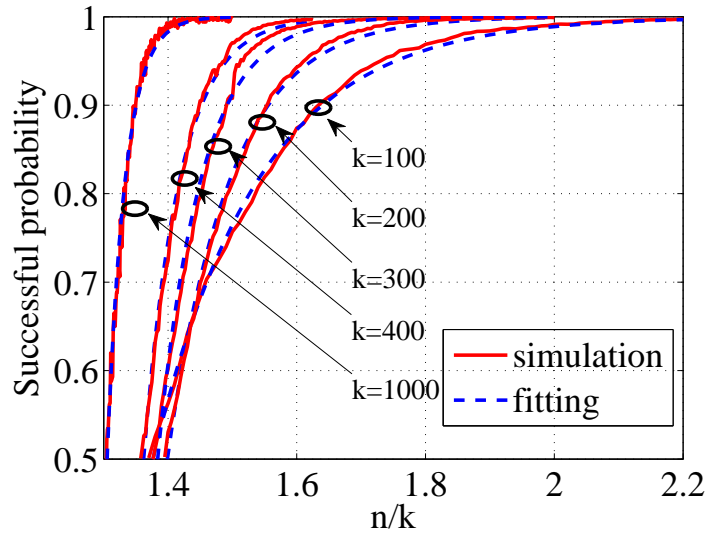

Fig. 4. Fitting results versus simulation results.

all the source symbols. In this example, if $P_{5}$ is missing, the decoding process will be terminated at Panel (b) since no more degree-1 encoded packets can be found after recovering $S_{2}$.

\subsection{LT Decodability}

As shown in the example in Section 4.1, the LT decoding process is probabilistic. Specifically, given a set of packets with a full-rank coding matrix, the decoding process could still fail with a certain probability. Moreover, the successful probability of decoding increases as the number of received packets $n$ increases. In this case, LT decodability, denoted by $f_{k}(n)$, is defined as the probability of successful decoding $k$ source symbols from a set of $n$ encoded packets $(n \geq k)$.

Existing research on characterizing the LT decodability has not resulted in a closed-form solution yet. In [35], the authors proposed a dynamic programming algorithm to calculate the LT decodability, with a computing complexity of $O\left(n^{3} \log ^{2} n\right)$. Another algorithm in [36] reduces the computing complexity to $O\left(k^{2} \log k\right)$. However, none of these previous research efforts resulted in a closed-form solution to the LT decodability, while relying on numerical evaluation using dynamic programming.

TABLE 2

Fitting results for $\alpha$ and $\beta$

\begin{tabular}{|c|c|c|c|c|c|}
\hline$k$ & 100 & 200 & 300 & 400 & 1000 \\
\hline$\alpha$ & 6.108 & 10.268 & 14.907 & 17.912 & 35.65 \\
\hline$\beta$ & 1.263 & 1.3315 & 1.3373 & 1.3235 & 1.286 \\
\hline$S S E$ & 0.003096 & 0.005643 & 0.01805 & 0.01113 & 0.02308 \\
\hline
\end{tabular}

In this paper, we derive an empirical model for the LT decodability, by leveraging the numerical evaluation techniques from [36]. Note that, in an LT-based cloud storage system, the decodability under $50 \%$ is of less engineering interest. As a result, we focus on the decodability over $50 \%$ in the rest of this paper. We use a two-step procedure to derive the empirical model for the LT decodability, as follows:
- Firstly, we plot the LT decodability as a function of the number of encoded packet, by using the numerical technique suggested in [36].

- Secondly, we use a generic function of $f_{k}(n)=$ $1-e^{-\alpha\left(\frac{n}{k}-\beta\right)}$ to curve fit the numerical results, where $\alpha$ and $\beta$ are two fitting parameters related to $k$.

We illustrate this approach in Figure 4. First, we obtain the simulated LT decodability for the cases of $k=100$, $k=200, k=300, k=400$ and $k=1000$ as illustrated with the solid curves. Second, using MATLAB's "Curve Fitting Tool" with a specific fitting function of $f_{k}(n)=1-e^{-\alpha\left(\frac{n}{k}-\beta\right)}$, we obtain the fitting results of $\alpha$ and $\beta$ together with the sum of square errors (SSE) in Table 2. Finally, we compare the LT decodability between the simulation results and the fitting results (the dotted curves) for $k=100,200,300,400$, and 1000, in Figure 4. It can be observed that the empirical model captures the simulation results with a decent accuracy.

In the rest of this paper, we will use this empirical model of the LT decodability as a basis for our optimization framework.

\section{File-Retrieval Delay Analysis}

In this section, we characterize the average fileretrieval delay for different request schemes in the presence of ambient traffic. To validate our analytical results, we run computer simulation written in Java using NetBeans 7.1. All the simulation results are obtained by averaging 1000 simulation instances. In simulations, we set that the length of packets in the ambient traffic follows an exponential distribution. Although it is a simplified model, the insights obtained with this simple model can be applied to guide practical system design. Table 3 lists the default values of some parameters in the simulator.

TABLE 3

Default values in Simulation

\begin{tabular}{|c|c|c|}
\hline Notation & Physical Meaning & Value \\
\hline$l$ & Length of packet for ambient traffic & 1024 Bytes \\
\hline$l_{L T}$ & Length of LT-coded packet & 1024 Bytes \\
\hline$r$ & Transmission rate & $800 \mathrm{kbps}$ \\
\hline
\end{tabular}

\subsection{Delay Analysis for One Stage Request Scheme}

We first investigate the file-retrieval delay $D_{1}(n)$ for $n$ LT packets in the one-stage request case. This delay consists of two parts as shown in Figure 5(a). The first part arises from the traffic arriving before the LT request and the second part arises from the traffic arriving after the LT request. In this analysis, we assume Poisson arrival process for ambient traffic and LT-coded packets. The first part only contains ambient traffic and it is treated as a classical M/G/1 queue ${ }^{2}$

2. More information on $M / G / 1$ queue can be found in [37]. 
This article has been accepted for publication in a future issue of this journal, but has not been fully edited. Content may change prior to final publication. Citation information: DOI 10.1109/TCC.2015.2430347, IEEE Transactions on Cloud Computing

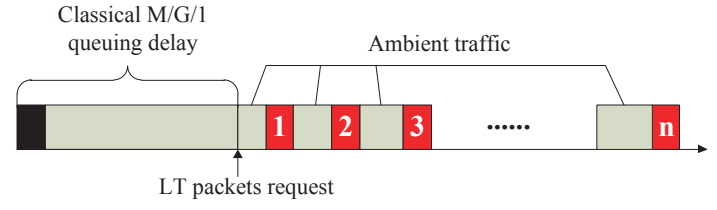

(a) One stage case

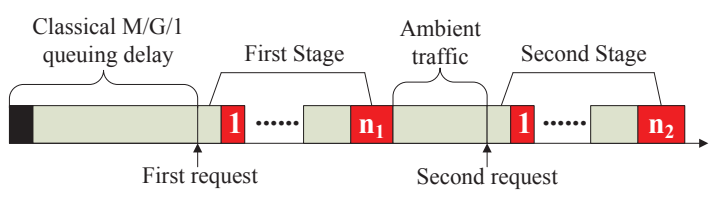

(b) Two stage case

Fig. 5. Components of delay.

with delay denoted by $W$. The second part can be further divided into two sub-parts. Firstly, it contains a constant which is the transmission time of $n$ LT packets, which can be derived as $\frac{n l_{L T}}{r}$. The rest is the time to process the ambient traffics which arrive at the portal during the inter-arrival time of each LT packet. This part is denoted by $T$. Thus, the delay $D_{1}(n)$ can be expressed as

$$
D_{1}(n)=W+\frac{n l_{L T}}{r}+T
$$

Taking an expectation on both sides, we obtain

$$
\begin{aligned}
\bar{D}_{1}(n) & =\bar{W}+\bar{T}+\frac{n l_{L T}}{r} \\
& =\frac{\lambda_{a}\left(\sigma^{2}+l^{2}\right)}{2 r^{2}\left(1-\frac{\lambda_{a} l}{r}\right)}+\frac{\lambda_{a} l}{r} \sum_{i=1}^{n} \tau_{i}+\frac{n l_{L T}}{r} \\
& =\Gamma+\frac{\lambda_{a} l}{r} \sum_{i=1}^{n} \frac{1}{i \theta}+\frac{n l_{L T}}{r} \\
& \simeq \Gamma+\frac{\lambda_{a} l}{r \theta}(\ln (n)+1)+\frac{n l_{L T}}{r},
\end{aligned}
$$

where $\Gamma$ is a constant when the parameters for the traffic are determined.

There are three terms in Eq. (5), $\Gamma$, the logarithm term and the linear term. In order to ensure the portal has a stable state, $\lambda_{a}$ should be less than 100 packets per second under our setting stated in Table 3 . Under this condition, the order of magnitude of $\Gamma$ is $10^{-3}$. The number of source symbols $k$ usually exceeds 100 which implies the number of requested packets $n$ is also larger than 100. Thus, compared to the linear term which has the order of magnitude equal to 10 or $10^{2}, \Gamma$ is negligible. If we assume that $\lambda_{a}$ and $\theta$ are comparable (e.g. $\left.\frac{\lambda_{a}}{\theta} \approx 1\right)$, the logarithm term has the order of magnitude equal to $10^{-2}$ which is also negligible. As a result, the linear term $\widetilde{D}_{1}(n)=\frac{n l_{L T}}{r}$ can be used to approximate $\bar{D}_{1}(n)$. In Figure 6, we plot the values of $\bar{D}_{1}(n)$ and $\widetilde{D}_{1}(n)$ for $n$ varying from 100 to 800 when $\lambda_{a}=30$ and $\theta=50$. We can observe that $\Gamma$ and the logarithm term contribute little to the retrieval delay. So it is reasonable to use $\widetilde{D}_{1}(n)$

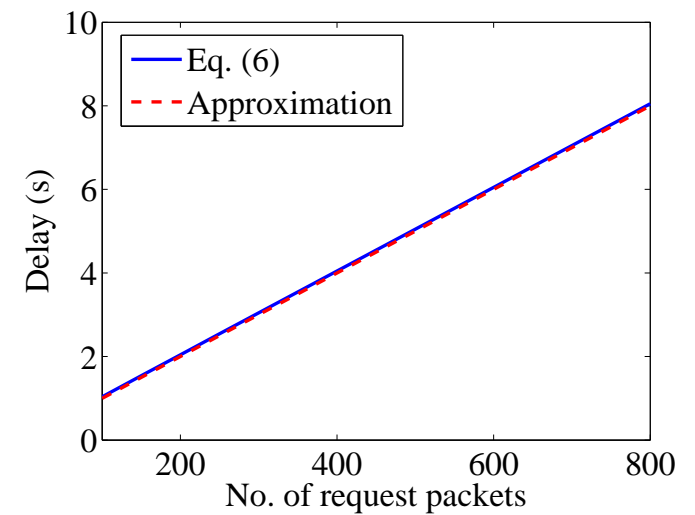

Fig. 6. Comparison between $\bar{D}_{1}(n)$ and $\widetilde{D}_{1}(n)$.

to approximate $\bar{D}_{1}(n)$. Similar results are found with different settings of $\lambda_{a}$ and $\theta$. For brevity, those results are not reported.

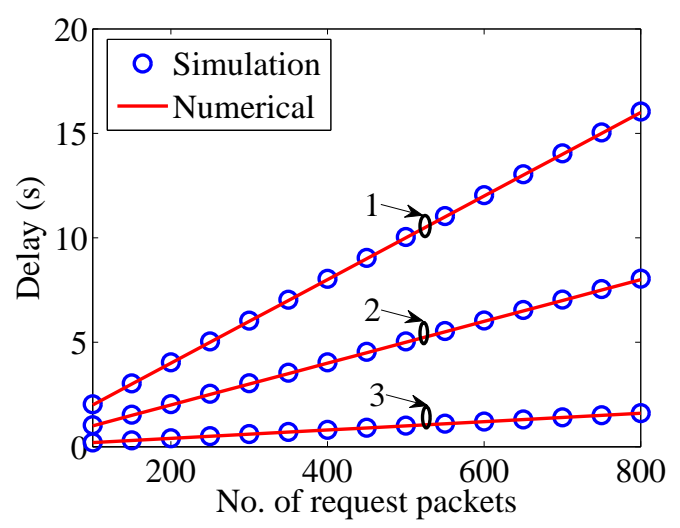

Fig. 7. Delay in one-stage request. $1: \lambda_{a}=10, \theta=25$; 2: $\lambda_{a}=30, \theta=50 ; 3: \lambda_{a}=60, \theta=50$.

In Figure 7, we plot the numerical retrieval delay $\widetilde{D}_{1}(n)$, compared with the simulation results, as a function of the number of request packets under different $\lambda_{a}$ and $\theta$ settings. Notice that the numerical results match the simulation results well, confirming that using $\widetilde{D}_{1}(n)$ to approximate Eq. (5) is applicable.

\subsection{Delay Analysis for Multiple Stage Request}

In this subsection, we first investigate the file-retrieval delay for the two-stage request scheme. The packet arrival process is illustrated in Figure 5(b).

Suppose in the first stage, the user requests $n_{1}$ LT encoded packets. The delay for the first stage is $D_{1}\left(n_{1}\right)$. After decoding these $n_{1}$ packets, if the user fails to decode the original file, it continues to request the $n_{2}$ packets. Here we assume that during the decoding process of $n_{1}$ packets, the transmission queue in the portal has already returned to the steady state. This assumption is reasonable since it does take some time for the user to determine if the $n_{1}$ LTencoded packets are decodable. Thus, the delay for 
This article has been accepted for publication in a future issue of this journal, but has not been fully edited. Content may change prior to final publication. Citation information: DOI 10.1109/TCC.2015.2430347, IEEE Transactions on Cloud Computing

the second stage is identical to the first stage except for the number of encoded packets the user requests. As a result, the overall file-retrieval delay for the two stage request case is given by

$$
\begin{aligned}
D_{2}\left(n_{1}, n_{2}\right) & =f_{k}\left(n_{1}\right) D_{1}\left(n_{1}\right) \\
& +\left(1-f_{k}\left(n_{1}\right)\right)\left(D_{1}\left(n_{1}\right)+D_{1}\left(n_{2}\right)\right) \\
& =D_{1}\left(n_{1}\right)+\left(1-f_{k}\left(n_{1}\right)\right) D_{1}\left(n_{2}\right),
\end{aligned}
$$

where $f_{k}(n)$ is the probability of successful decoding with $n$ LT encoded packets. Using the fitting results presented in Section 4.2, we can calculate $f_{k}(n)$ approximately. Taking expectation on both sides, we have

$$
\bar{D}_{2}\left(n_{1}, n_{2}\right)=\bar{D}_{1}\left(n_{1}\right)+\left(1-f_{k}\left(n_{1}\right)\right) \bar{D}_{1}\left(n_{2}\right) .
$$

We plot in Figs. 8-9 the average file-retrieval delay for the two-stage request scheme, as a function of the number of packets requested in the first stage, under different settings of $k, n$ and traffic loads. Notice that the numerical results match the simulation results well, regardless of the traffic loads. Moreover, in all results, the file-retrieval delay first decreases and then increases as the number of packets requested in the first stage increases. This consistent trend indicates a potential for file-retrieval delay minimization by choosing an appropriate number of packets requested in the first stage. We shall address this optimization problem in Section 6.

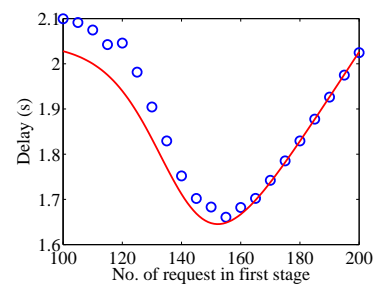

(a) $\lambda_{a}=10, \theta=25$

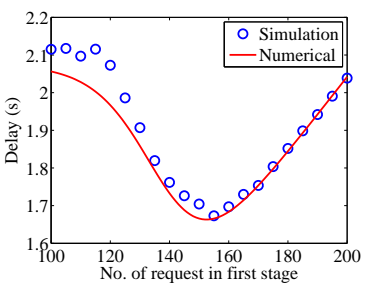

(c) $\lambda_{a}=30, \theta=50$

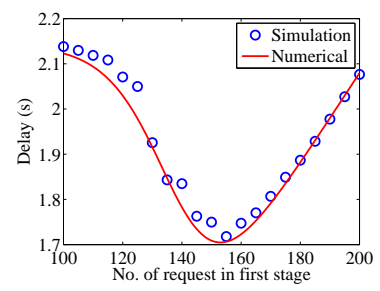

(b) $\lambda_{a}=30, \theta=25$

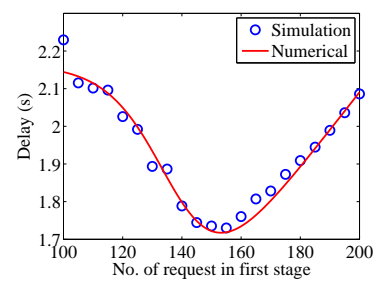

(d) $\lambda_{a}=60, \theta=50$
Fig. 8. Delay in two-stage request when $k=100$ and $n=200$ under different traffic loads. Both numerical and simulation results confirm that the minimal delay exists.

The result for the two-stage request scheme can be generalized into arbitrary $t$-stage request. Specifically, the delay expression for $t$-stage request can be defined

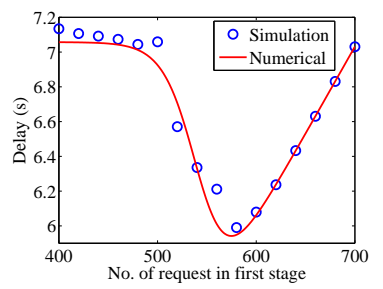

(a) $\lambda_{a}=10, \theta=25$

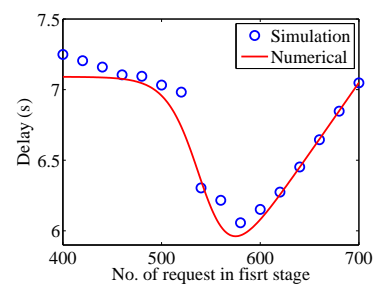

(c) $\lambda_{a}=30, \theta=50$

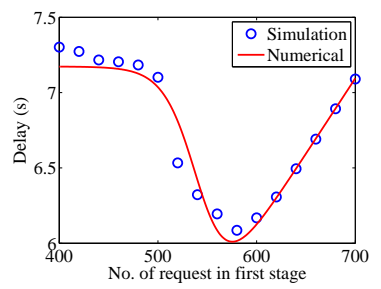

(b) $\lambda_{a}=30, \theta=25$

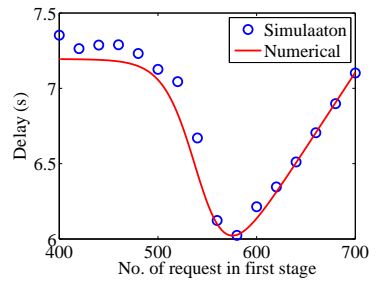

(d) $\lambda_{a}=60, \theta=50$
Fig. 9. Delay in two-stage request when $k=400$ and $n=700$ under different traffic loads. Both numerical and simulation results confirm that the minimal delay exists.

recursively,

$$
\begin{aligned}
D_{t}\left(n_{1}, n_{2}, \cdots, n_{t}\right) & =\left(1-f_{k}\left(n_{1}\right)\right)\left(D_{1}\left(n_{1}\right)\right. \\
& \left.+D_{t-1}\left(n_{2}, \cdots, n_{t}\right)\right) \\
& +f_{k}\left(n_{1}\right) D_{1}\left(n_{1}\right),
\end{aligned}
$$

where the initial values for $D_{1}, D_{2}$ are defined in Eq. (4) and Eq. (6).

In Figure 10 and 11, we plot the optimal delay comparison obtained by different request schemes for different number of original symbols under different traffic loads. Both figures show that the multiple stage request schemes outperform traditional onestage request. More specifically, the two-stage request scheme can reduce retrieval delay by $15 \%$ compared with the one-stage request. Whilst, the three-stage request scheme further reduces the delay $4 \%$ only. It is obvious that to find an optimal request in three-stage request is much more difficult than that in two-stage request and the improvement of three-stage request is not so significant. Consequently, we will only focus on finding an optimal two-stage request scheme in the rest of this paper.

\section{Optimal Scheduling for Packet Re- TRIEVAL}

In this section, we will investigate the optimal scheduling of packet requests to minimize the fileretrieval delay for two-stage request scheme.

The results in Figure 8 and Figure 9 suggest an optimal two-stage request scheme from Eq. (6). Another observation is that for a rational target successful probability (larger than 90\%), the optimal number of LT-coded packets requested in the first stage for a two-stage request scheme yields $f_{k}\left(n_{1}\right)>50 \%$. Thus, 
This article has been accepted for publication in a future issue of this journal, but has not been fully edited. Content may change prior to final publication. Citation information: DOI 10.1109/TCC.2015.2430347, IEEE Transactions on Cloud Computing

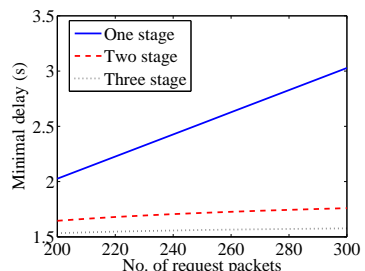

(a) $\lambda_{a}=10, \theta=25$

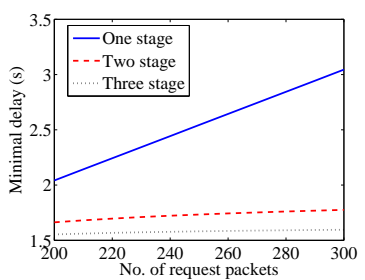

(c) $\lambda_{a}=30, \theta=50$

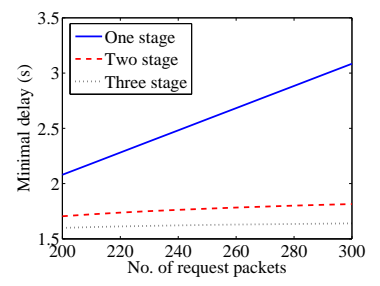

(b) $\lambda_{a}=30, \theta=25$

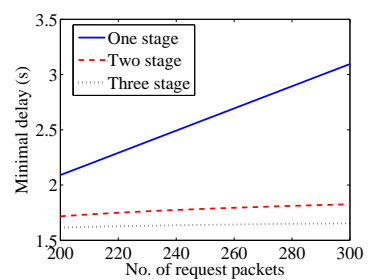

(d) $\lambda_{a}=60, \theta=50$
Fig. 10. Minimal delay comparison among one-stage request, two-stage request and three-stage request when $k=100$.

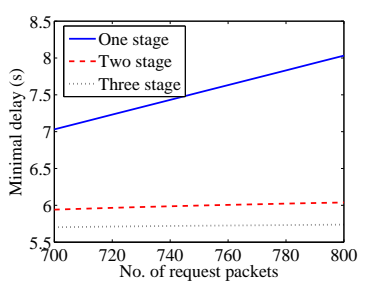

(a) $\lambda_{a}=10, \theta=25$

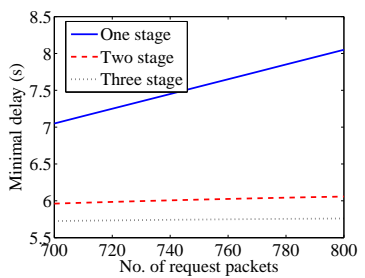

(c) $\lambda_{a}=30, \theta=50$

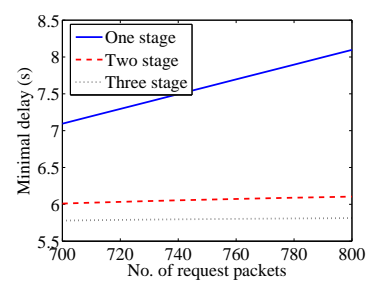

(b) $\lambda_{a}=30, \theta=25$

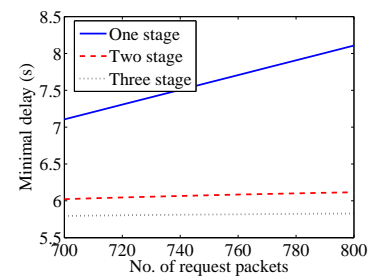

(d) $\lambda_{a}=60, \theta=50$
Fig. 11. Minimal delay comparison among one-stage request, two-stage request and three-stage request when $k=400$.

we can apply the fitting function for $f_{k}(n)$ to Eq. (6) and simplify the optimization problem presented in Section 3.3 for the two-stage request. For the convenience of discussion, we formalize the simplified optimization problem as:

$$
\begin{array}{cl}
\min & \bar{D}_{2}\left(n_{1}\right)=\bar{D}_{1}\left(n_{1}\right)+e^{-\alpha\left(\frac{n_{1}}{k}-\beta\right)} \bar{D}_{1}\left(n-n_{1}\right) \\
\text { s.t. } & k \beta \leq n_{1} \leq n, n_{1} \in \mathbb{N} .
\end{array}
$$

By solving the above optimization problem, we can determine the optimal number of packets requested in the first stage for a two-stage request scheme. Instead of finding the exact solution, we relax the integer constraint, and assume $n_{1}$ is a positive real number to solve the approximate problem without integer constraint. Note that the minimum $\bar{D}_{2}\left(n_{1}\right)$ without the integer constraint is a lower bound of the minimum $\bar{D}_{2}\left(n_{1}\right)$ with integer constraint.

We observed in Section 5.1 that $\bar{D}_{1}(n)$ can be approximated as $\frac{l_{L T}}{r} n$. Thus, $\bar{D}_{2}\left(n_{1}\right)$ in Eq. (8) is convex.

Now, taking differentiation with respect to $n_{1}$ on both sides of Eq. (8) and set $\frac{\partial \bar{D}_{2}}{\partial n_{1}}=0$, we get the equation $e^{\frac{\alpha}{k}\left(n_{1}-k \beta\right)}=\frac{\alpha}{k}\left(n-n_{1}\right)+1$. The solution to this equation is

$$
n_{1}^{*}=n+\frac{k}{\alpha}-\frac{k}{\alpha} W\left(e^{\alpha\left(\frac{n}{k}-\beta\right)+1}\right) .
$$

Here, $W(x)$ is Lambert function [38] which is defined as $x=W(x) e^{W(x)}$. Note that $W(x)$ cannot be expressed in terms of elementary functions, and thus calculating the real value of $W(x)$ is not an easy task. There exists literatures on designing algorithm to compute $W(x)$ [39], [40]. However, in this paper, we are more interested in the analytical bound of $W(x)$.

In [41], the authors proved the following bound of $W(x)$,

Lemma 1. For $x>1$ we have

$$
W(x) \geq \frac{\log x}{1+\log x}(\log x-\log \log x+1),
$$

with equality only for $x=e$.

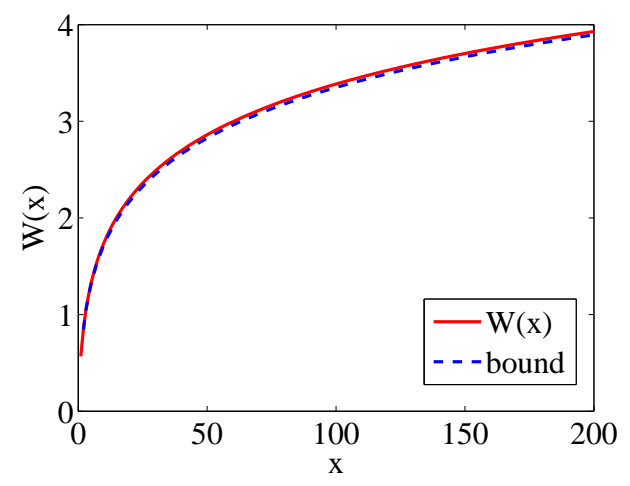

Fig. 12. Comparison between $W(x)$ and its bound.

Figure 12 shows the comparison between the true value of $W(x)$ and the lower bound in Eq. (10). It is clear that this lower bound is tight enough and we can use this bound to approximate $W(x)$.

By applying Eq. (10) on Eq. (9), we have

$$
\begin{aligned}
n_{1}^{*} & =n+\frac{k}{\alpha}-\frac{k}{\alpha} W\left(e^{\alpha\left(\frac{n}{k}-\beta\right)+1}\right) \\
& \approx k \beta+\frac{k}{\alpha} \frac{\left(\alpha\left(\frac{n}{k}-\beta\right)+1\right) \cdot \log \left(\alpha\left(\frac{n}{k}-\beta\right)+1\right)}{\alpha\left(\frac{n}{k}-\beta\right)+2} .
\end{aligned}
$$

It is trivial that $n_{1}^{*}>k \beta$, and

$$
\begin{aligned}
n_{1}^{*} & \leq k \beta+\frac{\alpha}{k} \log \left(\alpha\left(\frac{n}{k}-\beta\right)+1\right) \\
& \leq k \beta+\frac{k \alpha\left(\frac{n}{k}-\beta\right)}{\alpha} \\
& =n .
\end{aligned}
$$


Eq. (12) shows $n_{1}^{*} \leq n$. Thus, we confirm that $n_{1}^{*}$ is the optimal request in the first stage which minimizes the two-stage file-retrieval delay. In Figure 13, we show

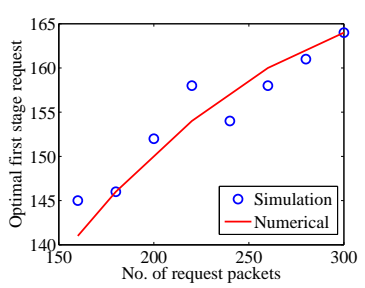

(a) $k=100$

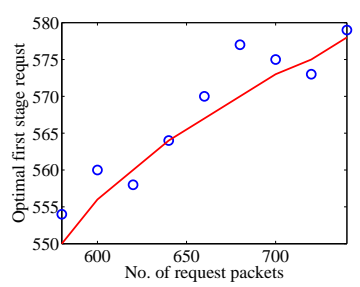

(c) $k=400$

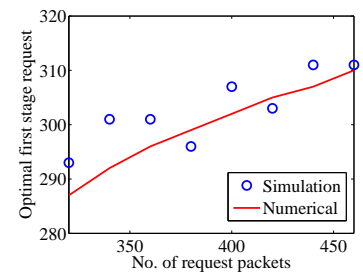

(b) $k=200$

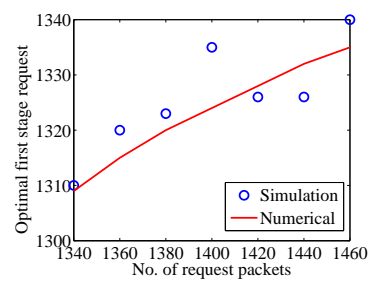

(d) $k=1000$
Fig. 13. Comparison of the optimal first stage request between simulation and approximation under different $k$.

the comparison of the optimal first stage request between simulation and approximation under different $k$. In the simulation, the traffic parameters $\lambda_{a}$ and $\theta$ are set to 30 packets per second and 50 packets per second respectively. The results confirm that the approximation of the optimal first stage request obtained from Eq. (11) is practical. In Eq. (11), the optimal firststage request is unrelated to traffic load (i.e. $\lambda_{a}, \theta$ ). When the number of original symbols $k$ is fixed (i.e. the parameters $a$ and $c$ are known), the optimal firststage request is determined. This is consistent with the results shown in Figure 8 and 9. This phenomena can be explained by the following reason. Compared with the LT encoded packets requested in each stage, the packets belonging to ambient traffic are negligible. The file-retrieval delay is mainly caused by the processing the requested LT encoded packets.

Based on Eq. (11), if we increase the target successful probability (i.e. increase the number of requested packets $n$ ), the optimal number of packets requested in first stage $n_{1}^{*}$ will be also increased. The ratio $\frac{n_{1}^{*}}{n}$ is decreased when $n$ is increased. We plot the ratio in Figure 14. This ratio dropping phenomena can be understood as follows. From the aspect of the decoding probability, every packet contributes in the decoding process. From Figure 4, we see an unequal contribution of each packet to the decoding probability. The contribution of an additional packet to the decoding probability is high when the probability stays at a lower level. As the probability progresses higher, the contribution of each additional packet drops. In other words, to make a same gain in the decoding probability, more packets are needed when the probability is at a higher level. Notice from Figure 6 that the delay in the one-stage request scheme increases linearly with the number of requested packets. Consequently, tradeoff exists between decodability and delay. Instead of requesting all the packets required by the target decoding probability at once, requesting a small portion of the number of packets needed in the first stage will generate a decoding probability that contributes most to the final decoding probability; while the marginal contribution from the second stage is relatively small. As the decodability increases, the contribution from the second stage becomes smaller. In other words, a proper number of packets requested in the first stage will give us a relatively high decoding probability. As a result, setting a very high decoding probability is not rational.

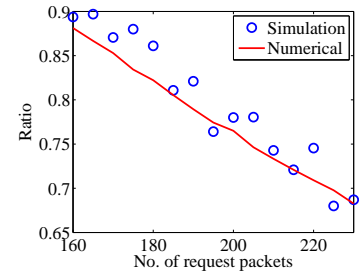

(a) $k=100$

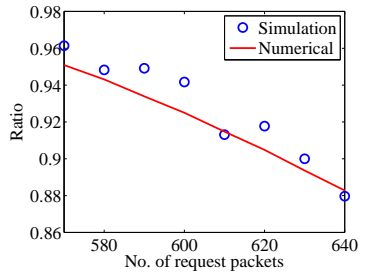

(b) $k=400$
Fig. 14. Optimal first stage request ratio when $k=100$ and $k=400$.

\section{Delay-Decodability Tradeoff}

We now investigate the fundamental tradeoff between the targeted decoding probability and the fileretrieval delay for different request schemes. This delay-decodability tradeoff is important since it helps users to make a rational request scheme depending on the requirement of their applications.

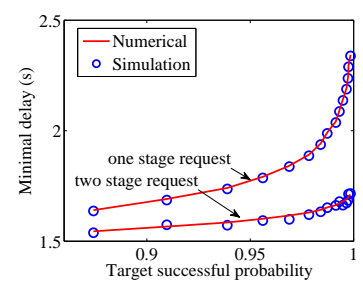

(a) $k=100$

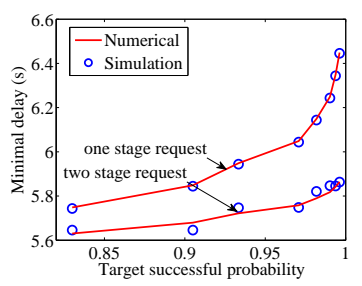

(b) $k=400$
Fig. 15. Tradeoff between decoding probability and delay when $k=100$ and $k=400$.

In Figure 15, we plot the delay-decodability tradeoff for $k=100$ and $k=400$ respectively. The numerical result is obtained by combining Eq. (6) and Eq. (11). Since traffic parameters do not have strong effect on the optimal retrieval delay (refer to the figures shown in Section. 5), we choose $\lambda_{a}=30, \theta=50$ as an example. As shown in Fig. 15, the delay for onestage request increases sharply when the decoding 
This article has been accepted for publication in a future issue of this journal, but has not been fully edited. Content may change prior to final publication. Citation information: DOI 10.1109/TCC.2015.2430347, IEEE Transactions on Cloud Computing

probability crosses $95 \%$. In comparison, for the twostage request scheme, the delay is consistently lower. This advantage reflects the gain from being able to decode in the first stage with an adequate instead of excessive number of LT-coded packets. Based on the reported delay-decodability tradeoff, users can choose an appropriate target of decoding probability, i.e. for some delay sensitive applications like stream video, a lower decoding probability can be adopted such that the average retrieval delay can be maintained under a certain threshold.

\section{General Traffic Model}

In Sections 6 and 7, we assume that the arrival process of ambient traffic follows Poisson process. In this section, we use a different traffic model to examine how our two-stage request scheme performs.

The Markov-modulated Poisson process (MMPP) [42] has been extensively used for modeling processes whose arrival rates vary randomly over time [43]. We use MMPP to model the ambient traffic here since it is more general than simple Poisson process and can capture some features to study burstiness of ambient traffic.

In our simulations, the MMPP traffic has arrival rates of $\lambda_{1}$ and $\lambda_{2}$ for the two phases, and an exponentially distributed rate switching duration with a mean value equal to $0.1 \mathrm{~s}$. We fix $\theta=50$ and vary the value of $\lambda_{1}, \lambda_{2}$ and $n$. The simulation results are shown in Figure 16.

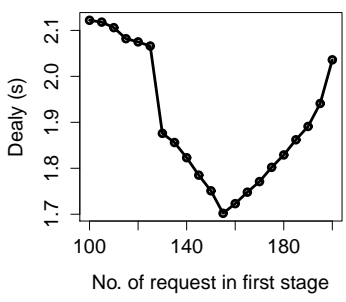

(a) $\lambda_{1}=10, \lambda_{2}=30$

(c) $\lambda_{1}=30, \lambda_{2}=60$

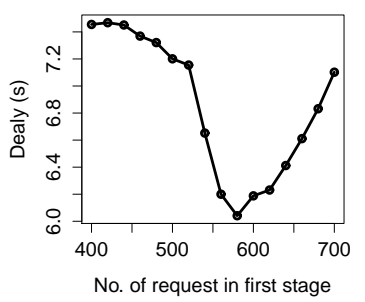

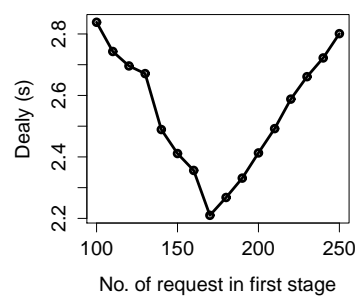

(b) $\lambda_{1}=10, \lambda_{2}=30$

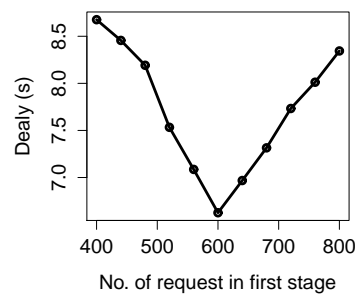

(d) $\lambda_{1}=30, \lambda_{2}=60$
Fig. 16. Simulation result of MMPP model. (a) $k=100$, $n=200$. (b) $k=100, n=250$. (c) $k=400, n=700$. (d) $k=400, n=800$.

Comparing with the simulation results shown in Figure 8 and Figure 9, we can see that our two-stage request scheme exhibits the same behavior even when the bursty MMPP traffic model is used. With a different decoding probability, the best first-stage request can still be found. Only the average delay is affected since more packets are needed to achieve a higher decoding probability. This observation confirms the optimal request in first stage in Eq. (11) which is independent of the property of ambient traffic. We believe that different models of ambient traffic only affect on the overall delay while using our two-stage request scheme can always give the minimal delay.

\section{Conclusion}

In this paper, we have investigated the problem of delay optimal file-retrieval under a distributed cloud storage system. The file is first LT-encoded and spread out into a list of distributed storage nodes. During retrieval, the user schedules the packet request in a multi-stage manner, with an objective to minimize the average file-retrieval delay. We developed an accurate model to characterize the average file-retrieval delay for different request strategies. Using this model, we derived an optimal two-stage request scheme for a given decoding probability. Both simulation and numerical results confirm that this optimal scheme can reduce the average delay dramatically. Our analysis offers a way for storage system operators to design an optimized storage retrieval scheme for LT-based distributed cloud storage systems.

\section{REFERENCES}

[1] Amazon S3. http://aws.amazon.com/s3/.

[2] EMC Atmos Online Storage. http://www.atmosonline.com/.

[3] L. Heilig and S. Voss, "A scientometric analysis of cloud computing literature," IEEE Transactions on Cloud Computing, vol. 2, no. 3, pp. 266-278, July 2014.

[4] J.-W. Lin, C.-H. Chen, and J. Chang, "Qos-aware data replication for data-intensive applications in cloud computing systems," IEEE Transactions on Cloud Computing, vol. 1, no. 1, pp. 101-115, Jan 2013.

[5] H. Weatherspoon and J. Kubiatowicz, "Erasure coding vs. replication: A quantitative comparison," Peer-to-Peer Systems, pp. 328-337, 2002.

[6] J. Hafner, "Weaver codes: highly fault tolerant erasure codes for storage systems," in Proc. the 4th conference on USENIX Conference on File and Storage Technologies. USENIX Association, 2005, pp. 16-16.

[7] J. Plank and L. Xu, "Optimizing cauchy Reed-Solomon codes for fault-tolerant network storage applications," in IEEE Int. Symp. Network Computing and Applications. IEEE, 2006, pp. 173-180.

[8] C. Huang, M. Chen, and J. Li, "Pyramid codes: Flexible schemes to trade space for access efficiency in reliable data storage systems," in IEEE Int. Symp. Network Computing and Applications. IEEE, 2007, pp. 79-86.

[9] F. Oggier and A. Datta, "Self-repairing homomorphic codes for distributed storage systems," in Proc. IEEE INFOCOM. IEEE, 2010, pp. 1215-1223.

[10] H. Xia and A. Chien, "Robustore: a distributed storage architecture with robust and high performance," in Proceedings of the 2007 ACM/IEEE conference on Supercomputing. ACM, 2007, p. 44.

[11] N. Cao, S. Yu, Z. Yang, W. Lou, and Y. T. Hou, "Lt codes-based secure and reliable cloud storage service," in INFOCOM, 2012 Proceedings IEEE. IEEE, 2012. 
This article has been accepted for publication in a future issue of this journal, but has not been fully edited. Content may change prior to final publication. Citation information: DOI 10.1109/TCC.2015.2430347, IEEE Transactions on Cloud Computing

[12] T. Benson, A. Akella, and D. Maltz, "Network traffic characteristics of data centers in the wild," in Proceedings of the 10th annual conference on Internet measurement. ACM, 2010, pp. 267-280.

[13] I. Reed and G. Solomon, "Polynomial codes over certain finite fields," Journal of the Society for Industrial and Applied Mathematics, vol. 8, no. 2, pp. 300-304, 1960.

[14] Symform. http://www.symform.com/.

[15] D. Connor, P. H. Corrigan, J. E. Bagley, and S. S. NOW, “Cloud storage: Adoption, practice and deployment," An Outlook Report from Storage Strategies NOW, 2011.

[16] M. Sardari, R. Restrepo, F. Fekri, and E. Soljanin, "Memory allocation in distributed storage networks," in Proc. Int. Symp. Information Theory ISIT. IEEE, 2010, pp. 1958-1962.

[17] D. Leong, A. Dimakis, and T. Ho, "Distributed storage allocation for high reliability," in IEEE Int. Conference on Communications (ICC). IEEE, 2010, pp. 1-6.

[18] A. Dimakis, P. Godfrey, Y. Wu, M. Wainwright, and K. Ramchandran, "Network coding for distributed storage systems," IEEE Transactions on Information Theory, vol. 56, no. 9, pp. 4539$4551,2010$.

[19] D. Leong, A. Dimakis, and T. Ho, "Distributed storage allocations for optimal delay," in Proc. Int. Symp. Information Theory ISIT, 2011.

[20] M. Luby, "LT codes," in Proc. 43rd Annual IEEE Symp. Foundations of Computer Science, 2002, pp. 271-280.

[21] R. Sandberg, D. Goldberg, S. Kleiman, D. Walsh, and B. Lyon, "Design and implementation of the sun network filesystem," in Proceedings of the Summer 1985 USENIX Conference, 1985, pp. 119-130.

[22] M. Nelson, B. Welch, and J. Ousterhout, "Caching in the sprite network file system," ACM Transactions on Computer Systems (TOCS), vol. 6, no. 1, pp. 134-154, 1988.

[23] P. Corbett and D. Feitelson, "The vesta parallel file system," ACM Transactions on Computer Systems (TOCS), vol. 14, no. 3, pp. 225-264, 1996.

[24] N. Nieuwejaar and D. Kotz, "The galley parallel file system," Parallel Computing, vol. 23, no. 4-5, pp. 447-476, 1997.

[25] P. Carns, W. Ligon III, R. Ross, and R. Thakur, "Pvfs: A parallel file system for linux clusters," in Proceedings of the 4th annual Linux Showcase E Conference-Volume 4. USENIX Association, 2000, pp. 28-28.

[26] F. B. Schmuck and R. L. Haskin, "Gpfs: A shared-disk file system for large computing clusters." in FAST, vol. 2, 2002, p. 19.

[27] M. Dunn and A. Reddy, A new I/O scheduler for solid state devices. Texas A\&M University, 2010.
[28] J. Ousterhout, P. Agrawal, D. Erickson, C. Kozyrakis, J. Leverich, D. Mazières, S. Mitra, A. Narayanan, G. Parulkar, M. Rosenblum et al., "The case for ramclouds: scalable highperformance storage entirely in dram," ACM SIGOPS Operating Systems Review, vol. 43, no. 4, pp. 92-105, 2010.

[29] S. P. Vanderwiel and D. J. Lilja, "Data prefetch mechanisms," ACM Computing Surveys, vol. 32, no. 2, pp. 174-199, 2000.

[30] V. Gupta, M. Harchol Balter, K. Sigman, and W. Whitt, "Analysis of join-the-shortest-queue routing for web server farms," Performance Evaluation, vol. 64, no. 9, pp. 1062-1081, 2007.

[31] M. Bjorkqvist, L. Chen, M. Vukolic, and X. Zhang, "Minimizing retrieval latency for content cloud," in Proc. IEEE INFOCOM, April 2011, pp. 1080-1088.

[32] B. Guan, J. Wu, Y. Wang, and S. Khan, "Civsched: A communication-aware inter-vm scheduling technique for decreased network latency between co-located vms," IEEE Transactions on Cloud Computing, vol. 2, no. 3, pp. 320-332, July 2014.

[33] Z. Wilcox-O'Hearn and B. Warner, "Tahoe: the least-authority filesystem," in Proceedings of the 4th ACM international workshop on Storage security and survivability, 2008, pp. 21-26.

[34] J. Pearl, "Fusion, propagation, and structuring in belief networks," Artificial intelligence, vol. 29, no. 3, pp. 241-288, 1986.

[35] R. Karp, M. Luby, and A. Shokrollahi, "Finite length analysis of LT codes," in Proc. Int. Symp. Information Theory ISIT, 2004, p. 39.

[36] E. Maneva and A. Shokrollahi, "New model for rigorous analysis of LT-codes," in Proc. Int. Symp. Information Theory ISIT, 2006, pp. 2677-2679.

[37] D. Gross, J. F. Shortle, J. M. Thompson, and C. M. Harris, Fundamentals of queueing theory. Wiley. com, 2013.

[38] R. Corless, G. Gonnet, D. Hare, D. Jeffrey, and D. Knuth, "On the lambert w function," Advances in Computational Mathematics, vol. 5, no. 1, pp. 329-359, 1996.

[39] F. N. Fritsch, R. E. Shafer, and W. P. Crowley, "Solution of the transcendental equation wew $=\mathrm{x}, "$ Commun. $A C M$, vol. 16, pp. 123-124, February 1973.

[40] D. A. Barry, P. J. Culligan-Hensley, and S. J. Barry, "Real values of the w-function," ACM Trans. Math. Softw., vol. 21, pp. 161171, June 1995.

[41] A. Hoorfar and M. Hassani, "Inequalities on the lambert $\mathrm{w}$ function and hyperpower function," J. Inequal. Pure and Appl. Math, vol. 9, no. 2, 2008.

[42] D. R. Cox, "Some statistical methods connected with series of events," Journal of the Royal Statistical Society, pp. 129-164, 1955.

[43] W. Fischer and K. Meier-Hellstern, "The markov-modulated poisson process (mmpp) cookbook," Performance Evaluation, vol. 18, no. 2, pp. 149-171, 1993. 
This article has been accepted for publication in a future issue of this journal, but has not been fully edited. Content may change prior to final publication. Citation information: DOI 10.1109/TCC.2015.2430347, IEEE Transactions on Cloud Computing

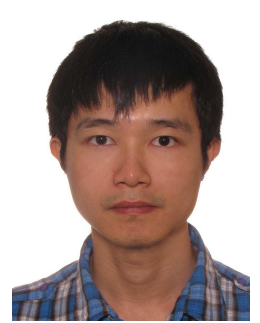

Haifeng Lu received his B.Eng degree in computer science and engineering from the University of Science and Technology of China in 2008. Since 2009, he has been a PhD student at School of Computer Engineering, Nanyang Technological University. His research interests include network coding, rateless coding, cloud computering. From June 2013 to July 2014, he was a project officer at Rapid-Rich Object Search (ROSE) Lab, NTU. Currently, he works at Alibaba Inc. as a senior security engineer.

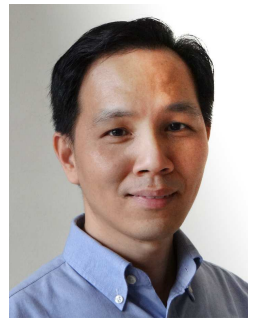

Chuan Heng Foh (S'00-M'03-SM'09) received his M.Sc. degree from Monash University, Australia in 1999 and Ph.D. degree from the University of Melbourne, Australia in 2002. After his PhD, he spent 6 months as a Lecturer at Monash University in Australia. In December 2002, he joined Nanyang Technological University as an Assistant Professor until 2012. He is now a Senior Lecturer at The University of Surrey. His research interests include protocol design and performance analysis of various computer networks, 5G networks, and data center networks. He has authored or coauthored over 100 refereed papers in international journals and conferences. He actively participates in IEEE conference and workshop organization, including the Internation Workshop on Cloud Computing Systems, Networks, and Applications (CCSNA) where he is a steering member. He is currently an Associate Editor for IEEE Access, IEEE Wireless Communications, and various other International Journals. $\mathrm{He}$ is also the chair of the Special Interest Group on Green Data Center and Cloud Computing under IEEE Technical Committee on Green Communications and Computing (TCGCC). He is a senior member of IEEE.

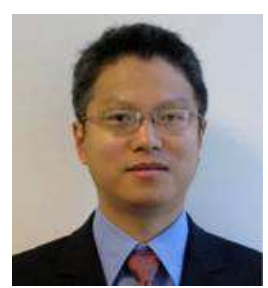

Yonggang Wen received the Ph.D. degree in electrical engineering and computer science from the Massachusetts Institute of Technology, Cambridge, MA, USA, in 2008. He is currently an Assistant Professor with the School of Computer Engineering, Nanyang Technological University, Singapore. Previously, he was with Cisco, San Jose, CA, USA, as a Senior Software Engineer and a System Architect for content networking products. He has also been a Research Intern with Bell Laboratories, Murray Hill, NJ, USA, Sycamore Networks, Chelmsford, MA, USA, and a Technical Advisor to the Chairman at Linear A Networks, Inc., Milpitas, CA, USA. His current research interests include cloud computing, mobile computing, multimedia networks, cyber security, and green ICT.

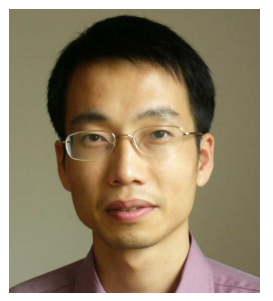

Jianfei Cai (S'98-M'02-SM'07) received his $\mathrm{PhD}$ degree from the University of MissouriColumbia. Currently, he is the Head of Visual \& Interactive Computing Division at the School of Computer Engineering, Nanyang Technological University, Singapore. His major research interests include visual information processing and multimedia networking. He has published over 100 technical papers in international conferences and journals. He has been actively participating in program committees of various conferences. He had served as the leading Technical Program Chair for IEEE International Conference on Multimedia \& Expo (ICME) 2012 and he currently sits in the steering committee of ICME. He was an invited speaker for the first IEEE Signal Processing Society Summer School on 3D and high definition / high contrast video process systems in 2011 . He is also an Associate Editor for IEEE Transactions on Circuits and Systems for Video Technology (T-CSVT), and a senior member of IEEE. 